\title{
A Semi-Classical Picture of the Charmonium-Hadron Interaction
}

\author{
D.A. Fogaça, M.S. Kugeratski, and F.S. Navarra \\ Instituto de Física, Universidade de São Paulo, C.P. 66318, 05389-970 São Paulo, SP, Brazil
}

Received on 15 August, 2003.

\begin{abstract}
In this work, we present calculations for the charmonium-hadron cross section. The hadron is represented by an external color field and the charmonium is represented by a small color dipole. Using high-energy approximations we compute the relevant cross sections, which agree with results obtained with other methods.
\end{abstract}

In the near future, data on charmonium production in heavy ion collisions at RHIC will be available. In order to understand them, a crucial ingredient is a good knowledge of the charmonium - hadron cross section. In the last years much work has been done along this line [1].

More than two decades ago, Peskin and Bhanot (BP) [2] showed that one could apply perturbative QCD to calculate the interactions between bound states of heavy quarks and light hadrons. The justification of their approach comes basically from the fact that the heavy quark mass provides a large energy scale. Later their results have been rederived in the context of the operator product expansion (OPE) [3] and more recently in terms of the QCD factorization theorem [4]. In all these works some nonperturbative input is needed, such as certain matrix elements involving gluon operators, the heavy quark wave function inside the bound state or moments of the gluon distribution function in the hadron. Moreover, most of the other existing calculations follow some nonperturbative approach, some of them using constituent quark wave functions [5], some others using effective lagrangians involving charm mesons in the strong coupling regime [6] (which, in many cases, need charm form factors [7]) and some others using the QCD vacuum expectation values (condensates), which appear naturally in the QCD sum rules approach [8]. All these treatments of the problem were devoted to low energy rections. However, it turns out that even at high energies $(\sqrt{s} \simeq 20 \mathrm{GeV})$ nonperturbative effects play a very important role [9].

In this work we calculate the charmonium - hadron cross section using the BP approach with simplifying assumptions, so as to render the calculations simple, analytic and to be able to treat more complex interactions, like those involving the $\chi$ states or the $(\bar{Q}-Q)_{8}-g$ pre-resonant (or color octet) state.

The starting point is the assumption that the charmonium (dipole) is small compared with the hadron (condenser). As a consequence, the $\bar{Q}-Q$ pair will interact mostly with the external color field but not with the (quark) sources. Moreover, the external color field is considered to have only low momentum components ("soft gluons") and thus is able to transfer only a small amount of energy, which will be barely enough to dissociate the bound state. The typical binding energy is $\epsilon \simeq 0.6 \mathrm{GeV}$. Therefore, in a first approximation

$$
\epsilon<<M
$$

where $M$ is the mass of the bound state $(M \simeq 3 \mathrm{GeV})$. The binding energy is also small compared to the collision energy

$$
\epsilon<<\sqrt{s}
$$

Approximation (1) justifies the use of quantum mechanical perturbation theory (the Born approximation) and approximation (2) justifies the use of the eikonal approximation, which, in this case, implies that the hadron follows a straight line trajectory and remains essentially undisturbed during the interaction.

With these assumptions we can write the interaction Hamiltonian as:

$$
H_{i n t}=g\left(T_{1}^{a} \vec{E}_{1}^{a} \vec{r}_{1}+\bar{T}_{2}^{b} \vec{E}_{2}^{b} \vec{r}_{2}\right)
$$

where $T^{a}\left(\bar{T}^{b}\right)$ are the generators of color group SU(3) in the fundamental (conjugate) representation. The index 1 and 2 represent the quark and antiquark respectively. $\vec{r}_{1}$ and $\vec{r}_{2}$ are the quark and antiquark coordinates in the charmonium rest frame. $\vec{E}_{1}^{a}$ and $\vec{E}_{2}^{b}$ are the chromoeletric fields generated by the hadron in motion (condenser) and "felt" by quark and antiquark in the bound state. They have to be Lorentz transformed to the charmonium rest frame, bringing to our calculation a Lorentz gamma factor, which is the source of the energy dependence $(\sqrt{s})$ of our results. We shall for the moment neglect the magnetic component, since it does not do any work on the charges and thus is not effective in the energy transfer.

We can represent this external field by:

$$
\begin{aligned}
\vec{E}\left(r_{e}, t\right)= & \gamma \vec{E}_{0} \exp \left(-\frac{\left(X-x_{e}\right)^{2}}{d^{2}}-\frac{\left(Y-y_{e}\right)^{2}}{d^{2}}\right) \\
& \exp \left(-\gamma^{2} \frac{\left[v t-z_{e}\right]^{2}}{d^{2}}\right)
\end{aligned}
$$

with $e=1,2$. $\mathrm{X}, \mathrm{Y}$ and $\mathrm{Z}$ are the hadron coordinates. $Z=v t$, because the hadron moves with velocity $\vec{v}$ along 
the $\mathrm{z}$ axis. We neglect the deflection of the hadron trajectory, because we are studying reactions in the nonperturbative regime, i.e., of the low momentum transfer. $\mathrm{X}$ and $\mathrm{Y}$ are related with the impact parameter $b$ by: $b^{2}=X^{2}+Y^{2}$.

Notice that, by simplicity, we choose one preferencial direction for the field, in this case, the $x$-axis.

Neglecting the CM motion, (3) can be rewritten as

$$
H_{\text {int }}=g\left(\frac{\lambda^{a}}{2} E_{1}^{a}+\frac{\lambda^{b^{T}}}{2} E_{2}^{b}\right)\left(\frac{x_{1}-x_{2}}{2}\right)
$$

Also for the sake of simplicity, from now on, in (5) we take $x_{1}-x_{2} \simeq a$, where $a$ is the typical separation between quark and antiquark. Initially the quark-antiquark pair is in a localized region of the space. The initial wave function of system has one spatial part and one color part.

$$
\Psi_{i n}=f\left(r_{1}, r_{2}\right) c_{n} d_{n}
$$

where $c_{n}$ and $d_{n}$, with $n=1,2,3$, are the initial color vector for quark and antiquark respectively, taken in a color singlet state. We choose

$$
f\left(r_{1}, r_{2}\right)=N_{i} \exp \left[-\frac{\vec{r}_{1}^{2}}{a^{2}}\right] \exp \left[-\frac{{\overrightarrow{r_{2}}}^{2}}{a^{2}}\right] \exp \left(-i \varepsilon_{i} t\right)
$$

where $\varepsilon_{i}$ is the charmonium initial energy and $N_{i}$ is a normalization constant. The initial wave function $\Psi_{i n}$ describes the confinement of quarks and also asymptotic freedom, as it allows the quarks to be independent inside the bag.

Under the action of the external field the initial wave function $\Psi_{i n}$ evolves to a final state $\Psi_{f}$ :

$$
\Psi_{f}=t\left(r_{1}, r_{2}\right) c_{j} d_{k}
$$

where $c_{j}$ and $d_{k}$, with $j, k=1,2,3$, are the quark and antiquark final color vectors and

$$
\begin{aligned}
t\left(r_{1}, r_{2}\right)= & N_{f} \exp \left(i \vec{p}_{1} \cdot \vec{r}_{1}\right) \\
& \exp \left(i \vec{p}_{2} \cdot \vec{r}_{2}\right) \exp \left(-i \varepsilon_{f} t\right)
\end{aligned}
$$

where $\vec{p}_{1}$ and $\vec{p}_{2}$ are the quark momenta; $\varepsilon_{f}$ is the final energy of the $c-\bar{c}$ pair and $N_{f}$ is a normalization constant. The $\Psi_{f}$ function represents the wave function of free particles. In the model, the quarks become free, inside the condenser and then suffer hadronization, which always occurs with probability one and does not change the cross section, which is thus given by

$$
d^{6} \sigma=2 \pi \int_{0}^{\infty} d b b \overline{\left|T_{f i}\right|^{2}} \cdot \frac{V}{\left(2 \pi^{3}\right)} d^{3} p_{1} \cdot \frac{V}{\left(2 \pi^{3}\right)} d^{3} p_{2} .
$$

The transition amplitude squared can be easily computed from (5), (6) and (8):

$$
\left|T_{f i}\right|^{2}=|<| \Psi_{f}\left|H_{i n t}\right| \Psi_{i n}>\left.\right|^{2}=T_{f i}^{*} T_{f i}
$$

Since color is not observed, we take the average of the all initial color states, and the sum of all final states:

$$
\left|T_{f i}\right|^{2} \rightarrow \overline{\left|T_{f i}\right|^{2}} \equiv \frac{1}{3} \sum_{n} \frac{1}{8} \sum_{a} \sum_{j} \sum_{k}\left|T_{f i}\right|^{2}
$$

As it is clear from (5), we will need to know the matrix element $\langle g E\rangle$. Following Kuzmenko and Simonov [10], we take it to be equal to $\langle g E>\simeq 1 \mathrm{GeV} / \mathrm{fm}$. Performing the integrations in (10), we plot the result for the proton - charmonium cross section in Fig. 1. The proton size of taken to be $d=0.8 \mathrm{fm}$ and the charmonium size equal to $a=0.2$ fm.

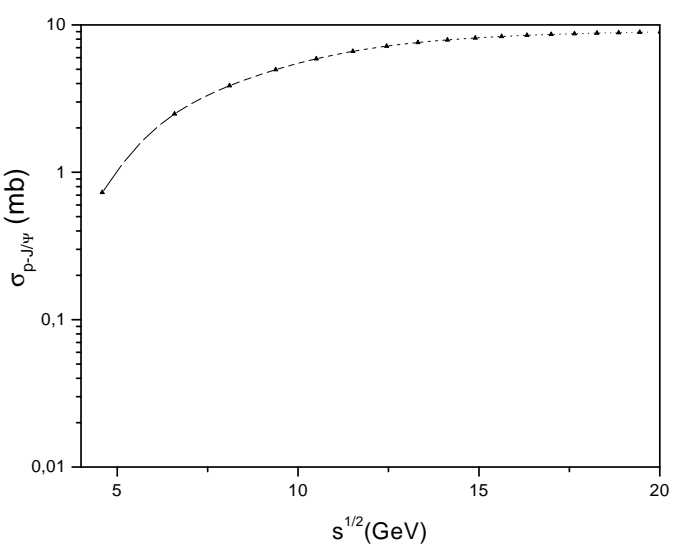

Figure 1. Proton-charmonium cross section as a function of $\sqrt{s}$.

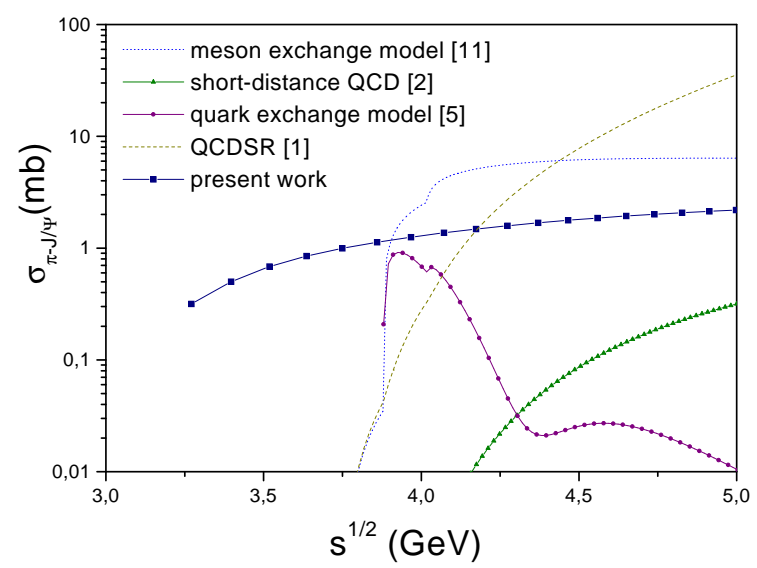

Figure 2. Pion-charmonium cross section as a function of $\sqrt{s}$.

In Figure 2, we show the results for the pion - charmonium cross section. As it can be seen, our curve agrees both with QCD sum rules results and with the results obtained with effective lagrangians, but is larger than the one obtained with the Bhanot-Peskin approach.

Given the conceptual similarity between our model and the $\mathrm{BP}$ one, it is somewhat surprising to find such a large discrepancy between both results (almost one order of magnitude). Both approaches depend on the projectile and target sizes $d$ and $a$ respectively. These are relatively well known quantities and they are certainly not responsible for the large 
discrepancies observed, which can be ultimately attributed to the different ways of incorporating the nonperturbative gluonic content of the hadron. In Refs. [3] and [4] the authors managed to rewrite the original chromoelectric field in terms of moments of the gluon distribution, whereas we have kept the quantity $<g E>$ untill the end of the calculation. This subject deserves further investigation.

\section{References}

[1] For a recent and comprehensive review, see F.O. Durães et al., Phys. Rev. C68, 035208 (2003).

[2] M.E. Peskin, Nucl. Phys. B156, 365 (1979) ; G. Bhanot and M.E. Peskin, Nucl. Phys. B156, 391 (1979).

[3] D.Kharzeev and H. Satz, Phys. Lett. B366, 316 (1996); B356, 365 (1995); B334, 155 (1994).

[4] Y. Oh, S. Kim, and S.H. Lee, Phys. Rev. C65, 067901 (2002).

[5] Cheuk-Yin Wong, E.S. Swanson, and T. Barnes, Phys. Rev. C65, 014903 (2002) .

[6] F.S. Navarra, M. Nielsen, and M.R. Robilotta, Phys. Rev. C64, 021901 (R) (2001) and references therein.
[7] F.S. Navarra, M. Nielsen, M. E. Bracco, M. Chiapparini, and C.L. Schat, Phys. Lett. B489, 319 (2000); F.O. Durães et al., Phys. Lett. B498, 169 (2001); M.E. Bracco et al., Phys. Lett. B521, 1 (2001); F.S. Navarra, M. Nielsen, and M.E. Bracco, Phys. Rev. D65, 037502 (2002); R.D. Matheus et al., Phys. Lett. B541, 265 (2002).

[8] F.S. Navarra, M. Nielsen, R.S. Marques de Carvalho, and G. Krein, Phys. Lett. B529, 87 (2002); M. Nielsen et al., Braz. Jour. Phys. 33 , 316 (2003); F.O. Durães et al., Phys. Lett. B564, 97 (2003).

[9] H.G. Dosch et al., Phys. Lett. B466, 363 (1999).

[10] V. Kuzmenko and Y.A. Simonov, Phys. Lett. B494, 81 (2000).

[11] S. G. Matinyan and B. Müller, Phys. Rev. C58, 2994 (1998); K. Haglin, Phys. Rev. 61, 031902 (2000); Z. Lin and C.M. Ko, Phys. Rev. 62, 034903 (2000); Y. Oh, T. Song, and S.H. Lee, Phys. Rev. 63, 034901 (2001); A. Sibirtsev, K. Tsushima, and A.W. Thomas, Phys. Rev. 63, 044906 (2001); W. Liu, C. M. Ko, and Z.W. Lin, nucl-th/0107058 (2001). 